\title{
DESENVOLVIMENTO DE NOVOS SERVIÇOS, ORIENTAÇÃO PARA O CLIENTE, COMPETÊNCIAS PROFISSIONAIS E RETENÇÃO DE CLIENTES EM SERVIÇOS DE SAÚDE
}

\author{
THE DEVELOPMENT OF NEW SERVICES, CUSTOMER ORIENTATION, PROFESSIONAL SKILLS AND \\ CUSTOMER RETENTION IN HEALTH SERVICES
}

(iD) Luciene Eberle ${ }^{1}$

Gabriel Sperandio Milan ${ }^{2}$
${ }^{1}$ Universidade de Caxias do Sul Doutora em Administração pela Associação Ampla PUCRS - Pontifícia Universidade Católica do Rio Grande do Sul e UCS - Universidade de Caxias do Sul (2014). leberle@ucs.br

${ }^{2}$ Pós-Doutor em Administração na área de Marketing pela

UFRGS - Universidade Federal do Rio Grande do Sul (2016). gsmilan@unisinos.br

\begin{abstract}
Resumo
Em mercados cada vez mais competitivos, o Desenvolvimento de Novos Serviços (DNS) assume um papel primordial na criação de desempenho e vantagem competitiva para as prestadoras de serviços. No presente estudo, foi elaborado e testado um Modelo Teórico, considerando os construtos Orientação para o Cliente, Competências Profissionais e Retenção de Clientes como consequentes do DNS. A amostra foi composta por clientes dos planos de saúde particular e consistiu em 247 casos válidos. Para a análise dos dados, foram utilizadas técnicas estatísticas multivariadas, através da modelagem de equações estruturais. Os resultados encontrados evidenciaram relações significativas entre os resultados de DNS, que afetam o sucesso dos novos serviços lançados pelo operador do plano de saúde, a partir da percepção dos clientes. Os novos serviços podem resultar em maior desempenho organizacional e maior competitividade para os provedores de serviços de saúde.
\end{abstract}

Palavras-chave: Desenvolvimento de Novos Serviços (DNS). Orientação para o Cliente. Competências Profissionais. Retenção de Clientes. Saúde.

\begin{abstract}
As the competitiveness in all markets grows on a daily basis, the Development of New Services (DNS) plays a key role in creating performance and competitive advantage for service providers. In the present study, a Theoretical Model was elaborated and tested, considering the Client Orientation, Professional Skills and Customer Retention as consequent constructs of the DNS.The sample comprised clients of the private health plans and consisted of 247 valid cases. For the analysis of the data, multivariate statistical techniques were used, through the modeling of structural equations. The results found evidenced the significant relationships between the DNS results, which affect the success of the new services launched by the health plan operator, based on the clients' perception. The new services can result in greater organizational performance and greater competitiveness for health service providers.
\end{abstract}

Keywords: New Services Development (NSD). Customer orientation. Professional skills. Customer retention. Health. 


\section{Introdução}

As bases conceituais que nortearam a investigação por muitos anos sobre a inovação em serviços e o Desenvolvimento de Novos Serviços (DNS) sofreram modificações (Papastathopoulou \& Hultink, 2012). O mundo atual globalizado exige perspectivas claras sobre os valores dos serviços, levando em consideração o aumento da criação de redes e interconexão de clientes com as empresas (Dominguez-Péry, Ageron \& Neubert, 2013). Diante disso, a literatura aponta a necessidade de novos estudos que abordam o Desenvolvimento de Novos Serviços (DNS), focando na qualidade elevada e no envolvimento com o cliente, como um fator chave para as empresas, pois contribui para o sucesso da inovação na economia de serviços (Snyder et al., 2016).

O DNS é um esforço que vai além da singularidade de uma nova oferta, pois abrange o sistema de entrega do serviço como um todo, a tecnologia, os processos utilizados e a interface com cliente (Jong \& Vermeulen, 2003). Em complemento, Gremyr et al. (2014) destacam que os processos de DNS são mais estruturados e precisam abordar o papel do cliente como co-produtor, onde simplesmente ajustar as etapas do Desenvolvimento de Novos Produtos (DNP) não é vista como confiável, dada a natureza fundamentalmente diferente das ofertas de serviços.

O desenvolvimento de serviços inovadores, portanto tornou-se uma importante fonte de diferenciação e vantagem competitiva (Storey \& Hull, 2010). Netse sentido, Gebauer, Gustafsson e Witell (2011) salientam que a inovação em serviços continua sendo uma prioridade fundamental nas pesquisas de serviços porque enfatiza uma vantagem competitiva sustentável, que depende do desenvolvimento e introdução de novos serviços de forma estratégica (Witell et al., 2017). Porém, é importante ressaltar que, embora o setor de serviços tenha crescido substancialmente do ponto de vista econômico, os gerenciamentos da inovação e do DNS continuam sendo um dos desafios mais importantes, estrategicamente, deste setor (O’Cass, Song \& Yuan, 2013).

A importância da superação de lacunas do conhecimento sobre a inovação e o DNS, se evidencia no complexo segmento da saúde, no qual, cada vez mais, têm se organizado mercadologicamente (Costa, 2016). Outro aspecto de destaque neste ser, é que as inovações por meio do desenvolvimento de novos serviços em saúde, exigem o envolvimento da gerência e da equipe de linha de frente para serem bem-sucedidas (Birken et al., 2013; Bokhour et al., 2018).

Ao se fazer um panorama das operadoras de planos de saúde, salienta-se que no Brasil, ao fim do primeiro trimestre de 2019, havia 471 milhões de vínculos de beneficiários na saúde suplementar. Porém, em comparação com o ano de 2018, houve uma redução de 196,4 mil vínculos no segmento médico-hospitalar (ANS, 2019), devido à retração na atividade econômica do país, o que levou a uma diminuição do número de beneficiários nos planos de saúde (Almeida \& Guerra, 2019).

Diante disso, Papastathopoulou e Hultink (2012) salientam que, há um gap para o desenvolvimento de pesquisas futuras em relação de como as prestadoras de serviços possam criar mais sinergia nos novos serviços, aproveitando melhor os recursos, habilidades e experiências. Outra 
oportunidadepara novos estudos que os autores sinalizam é a criação de um modelo de inovação integrativo, levando em consideração o envolvimento dos clientes nas trocas relacionais.

Levando isso em consideração, testou-se as relações entre os construtos, orientação para o cliente, competências profissionais e retenção de clientes, com a intenção de definir com maior precisão os consequentes que impactam no DNS. Neste horizonte, a literatura aponta que o aumento da orientação para o mercado, leva ao aprimoramento das capacidades de conexão com os clientes, fortalecendo os relacionamentos de longo prazo, enfatizando o aumento das habilidades dos profissionais no desenvolvimento e lançamento de novos produtos e/ou serviços, reforçando a capacidade da continuidade dos relacionamentos com os clientes (Sampaio, Perin\& Ferreira, 2008).

Com base nesta discussão, o objetivo central deste estudo foi o de investigar os construtos Orientação para o Cliente, Competência Profissional e Retenção de Clientes como conseqüentes do Desenvolvimento de Novos Serviços (DNS), no contexto de uma operadora de planos de saúde, no segmento B2C - Business-to-Consumer.

\section{Modelo teórico e hipóteses de pesquisa}

É importante salientar que as práticas do DNS capturam como as empresas gerenciam o processo de desenvolvimento de novos serviços (Biemens et al., 2016; Jaakkola et al., 2017), destacando os processos mais formalizados como um fator de sucesso (Kuester et al., 2013). Outra função importante, diz respeito à forma como os dadod ou as informações dos clientes são coletadas, destacando o envolvimento do cliente no processo de criação de novos serviços (Edvardsson et al., 2012; Papastathopoulou \& Hultink, 2012).

Verifica-se, também, que há um crescimento relevante no número de artigos publicados em torno do desenvolvimento de novos serviços nos últimos anos. Porém, as tentativas de desenvolver a lista dos construtos-chaves das práticas atinentes ao DNS são limitadas e os apontamentos da literatura são, predominantemente, baseados em discussões conceituais (Graciola et al., 2017; Carbonell \& Escudero, 2014).

Sendo assim, a orientação para o cliente, é o primeiro construto a ser testado, como consequente do DNS. A orientação para o cliente é o foco principal para o relacionamento de qualquer empresa com o mercado onde está inserida (Kohly \& Jaworkis, 1990; Frambach, Fiss \& Ingenbleek, 2016). O termo orientação para o cliente significa foco no atendimento aos interesses, necessidades e expectativas dos clientes na entrega de serviços adequados e personalizados (Wang, Zhao \& Voss, 2016).

Considerando a ênfase tradicional do marketing, a orientação para o cliente visa ajudar os clientes a tomarem decisões que se encaixam não só nas necessidades imediatas, mas também, destina-se a satisfazer as necessidades de longo prazo, além de ajudar para que as empresas alcancem um crescimento sustentável. Dessa forma, Ludwiczak (2016) apoia o desenvolvimento da abordagem 
da orientação para o cliente e destaca que a gerência dos serviços de saúde deve, constantemente, analisar as opiniões de seus clientes (pacientes) para definir as melhorias que envolvem a prestação de serviços, visando atingir níveis de qualidade de excelência. Complementando tal raciocínio, Sindakis (2015) destaca que no mercado da prestação de serviços da saúde suplementar, o qual é altamente competitivo, as empresas precisam desenvolver novos e bem-sucedidos serviços para atender às necessidades, demandas ou expectativas dos clientes (usuários dos serviços).

Outro construto testado, refere-se às competências profissionais que são definidas como uma tendência ou predisposição dos funcionários para atender às necessidades dos clientes em um contexto de trabalho e que reflete diretamente no desempenho organizacional (Bruno, Dell'Aversana \& Zunino, 2017), sustentando uma performance superior na prestação de serviços (McCoy, LevettJones \& Pitt, 2013). O pessoal de contato direto (front office) com o cliente tem uma grande interferência na formação das expectativas, gerenciamento e controle das experiências dos clientes, além de moldar a avaliação geral do serviço (Tajeddini, 2011).

Reforçando esta ideia, Ludwiczak (2016) afirma que o sucesso das organizações de serviços com base na satisfação de clientes, depende, em grande escala, da satisfação dos funcionários. No caso da prestação de serviços de saúde, isso significa que o valor entregue aos clientes (pacientes) depende da competência e do envolvimento dos profissionais de saúde.

Sendo assim, alguns autores sugerem que devem ser desenvolvidos estudos que testem o impacto da orientação para o cliente nas competências profissionais nos encontros dos serviços (Niedenthal \& Brauer, 2012). Contudo, Brach et al. (2015) destacam que, a empatia dos funcionários, as suas experiências e a sua confiabilidade afetam as suas competências e se relacionam diretamente com o fortalecimento dos relacionamentos dos clientes com o provedor de serviços. Porém, deve-se levar em conta que os funcionários da linha de frente se diferem em suas habilidades individuais para satisfazer às necessidades dos clientes (Groth, Hennig-Thurau \& Walsh, 2009; Wu et al., 2015).

As competências profissionais indicam a vontade e a capacidade dos funcionários a prestar serviços de excelência e, consequentemente, os clientes percebem que a empresa responde às suas necessidades, fazendo com que os mesmos, realizem avaliações positivas sobre o provedor de serviços (Wu et al., 2015). Aliás, Brach et al. (2015) evidenciaram que a competência profissional demonstra um efeito positivo sobre a retenção de clientes; enquanto que Wu et al. (2015) destacam que outros estudos precisam testar esta relação com mais precisão.

Outra relação testada foi o impacto da orientação para o cliente na retenção de clientes. Isto se justifica porque, o propósito básico da orientação para o cliente é antecipar e satisfazer as necessidades dos clientes, com intuito de motivá-los para recomprar da mesma empresa, com a intenção de minimizar os riscos percebidos em comprar de outro fornecedor de serviços e por ter recebido valor superior, o que conduz positivamente a retenção dos clientes (Rao et al., 2011). 
Em se tratando da retenção de clientes, Milan, Eberle e Bebber (2015) comentam que a retenção de clientes é um tema chave para as empresas prestadoras de serviços, uma vez que se refere ao estabelecimento de relações de longo prazo com os clientes, por criar e entregar valor adicional (Hillebrand, Nijholt \& Nijssen, 2011). Em acréscimo, Díaz (2017) afirma que a retenção de clientes tem sido explicada na literatura como um resultado de diversos fatores que decorrem da experiência de consumo.

Segundo Arnold, Fang e Palmatier (2011), uma orientação voltada à retenção de clientes implica na obtenção de informações sobre o foco da alocação de recursos para o gerenciamento dos relacionamentos com os clientes baseados em seu valor no longo prazo, visando à manutenção dos clientes existentes. Em complemento, um elevado nível de retenção de clientes, provavelmente, conduzirá a um conhecimento homogêneo da carteira de clientes, permitindo um aperfeiçoamento dos relacionamentos entre cliente e provedor de serviços. No caso do setor de saúde, no qual os "pacientes" são clientes, a orientação para o cliente é definida como a capacidade do provedor de serviços em ajustar seus atendimentos, de uma maneira que reflita e atendas as necessidades específicas dos clientes (Ludwiczak, 2016).

Além disso, Amidu e Aluko (2007) salientam que o tempo de cliente, diz respeito à importância da continuidade dos clientes nos negócios. Por isso, pode-se inferir que quanto mais alto o tempo como cliente, maior será a intenção de permanecer retido, tendo uma tendência em continuar fazendo negócios com o mesmo provedor de serviços, mesmo que possa acontecer alguma falha no serviço (Neto, Souza \& Souki, 2011; Dagger \& David, 2012). Sendo assim, balizada pela proposição dos autores, espera-se que a diferença entre o tempo como cliente alto e baixo em relação à orientação para o cliente, possam potencializar a retenção de clientes.

Portanto, o modelo teórico testado verifica as relações dos construtos Orientação para o Cliente, Competências Profissionais e Retenção de Clientes, como conseqüentes do Desenvolvimento de Novos Serviços. O Modelo Teórico proposto é apresentado na Figura 1, bem como as respectivas hipóteses de pesquisa. 
Figura 1 - Modelo Teórico proposto

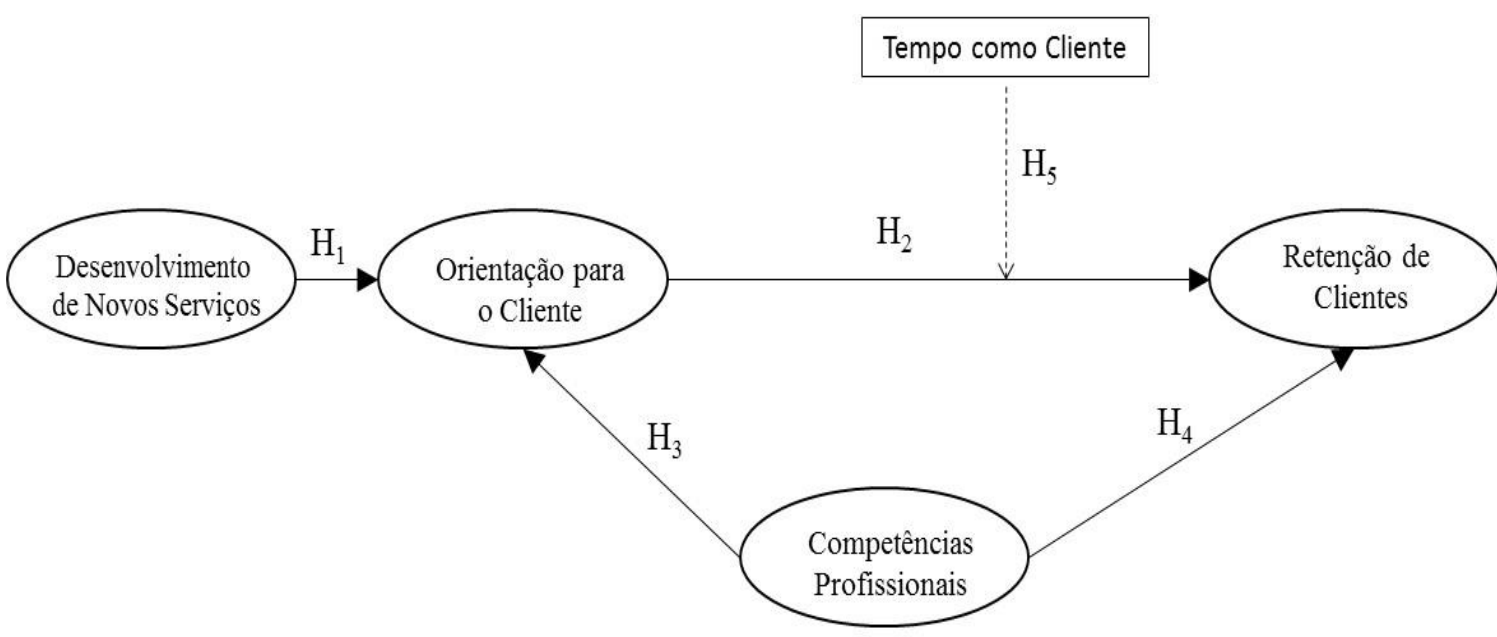

Efeito Moderador

Fonte: Autores

Portanto, as relações hipotetizadas e intrínsecas ao Modelo Teórico proposto são respectivamente:

H1: O desenvolvimento de novos serviços influencia positivamente a orientação para o cliente no provedor de serviços.

H2: A orientação para o cliente influencia positivamente a retenção de clientes.

H3: A competência profissional influencia positivamente a orientação para o cliente.

H4: A competência profissional influencia positivamente a retenção de clientes.

$\mathrm{H}_{5}$ : O tempo de cliente modera positivamente a relação entre a orientação para o cliente e a retenção de clientes.

\section{Método de pesquisa}

No presente estudo, aplicou-se uma pesquisa de levantamento (survey) com corte transversal (Malhotra, Birks \& Wills, 2012). A análise das relações propostas no Modelo Teórico, por sua vez, foi realizada através da Modelagem de Equações Estruturais. Para a operacionalização dos construtos, a escala definida para os construtos testados no presente estudo foi do tipo Likert de sete pontos, tendo em seus extremos "1. Discordo Totalmente" a "7. Concordo Totalmente, conforme Figura 2. 
Figura 2 - Operacionalização dos construtos

\begin{tabular}{|c|c|c|}
\hline Construtos & Itens de Escala & Autores \\
\hline $\begin{array}{c}\text { Desenvolvimento de Novos Serviços } \\
\text { (DNS) }\end{array}$ & DNS_1 a 6 & $\begin{array}{c}\text { Hu, Hong \& Sung (2009) } \\
\text { Kim \& Atuahene-Gima (2010) }\end{array}$ \\
\hline Orientação para o Cliente & OC_1 a 8 & Ordanini \& Parasuraman (2011) \\
\hline Competências Profissionais & CP_1 a 4 & Wu et al. (2015) \\
\hline Retenção de Clientes & RC_1 a 5 & Milan, Eberle \& Bebber (2015) \\
\hline
\end{tabular}

Fonte: Autores.

Uma vez elaborado e estruturado o questionário de pesquisa, foi procedida à validação de conteúdo, também conhecida como validade nominal (Malhotra, Birks \& Wills, 2012), sendo que o questionário foi submetido a dois experts da área. A seguir, foi aplicado o pré-teste, sendo que o instrumento de coleta de dados foi submetido a 15 respondentes que possuem o plano de saúde, da categoria familiar, da operadora em estudo. É importante salientar que os casos do pré-teste não foram incorporados à amostra final.

\subsection{População-alvo e definição da amostra}

A população determinada para essa pesquisa incluiu os clientes (B2C) de uma operadora de planos de saúde, residentes em uma cidade do Estado do Rio Grande do Sul, Brasil. Foi também definido que os respondentes do questionário deveriam ser os responsáveis pela aquisição do plano de saúde, na categoria planos de saúde familiares (particulares). Para isso, foi determinada uma amostra por conveniência, dentre os componentes alvos da população (Malhotra, Birks \& Wills, 2012). Sendo assim, a amostra final totalizou 247 casos válidos.

Em se tratando da caracterização da amostra, foram coletadas informações a respeito do perfil dos respondentes. Em relação ao nível de renda, 163 (65,99\%) respondentes recebem até três salários mínimos. Quanto ao tempo em que os respondentes são clientes da operadora de planos de saúde, verifica-se que há uma tendência pela manutenção do relacionamento com o provedor de serviços, indicando uma possível evidência de incidência da retenção dos clientes. O tempo de cliente teve uma média de 7,98 anos.

É válido destacar que foi incluido na amostra apenas questionários com dados completos, seguindo o procedimento para os casos omissos, conhecido como listwise deletion. Diante disso, a partir dos 255 questionários coletados, 5 casos foram excluídos.

O passo seguinte na análise dos dados foi à detecção dos outliers, por meio do Z scores (Hair Jr. et al., 2010). A partir das observações atípicas univariadas, não houve nenhum caso para ser eliminado. Os outliers multivariados foram investigados por meio do cálculo da distância de Mahalanobis $\left(D^{2}\right)$, considerando valores superiores 3 ou 4, com significância de $p<0,005$ e grau de liberdade $(g l=23)$, onde 3 casos foram eliminados, resultando em uma amostra final com 247 casos 
válidos. Em relação ao modelo, aplicou-se o método de estimação de máxima verossimilhança (MLE) e, a matriz escolhida para a entrada dos dados, neste estudo, foi a matriz de covariância (Byrne, 2016).

\section{Análise dos resultados}

\subsection{Validade individual dos construtos}

A seguir, realizou-se a validação individual dos construtos (Kline, 2015). O procedimento utilizado para a aferição da unidimensionalidade foi a Análise Fatorial Exploratória (AFE), onde os resultados foram satisfatórios, com um índice de 0,000 no teste de Bartlett Spherecity e 0,968 no teste de Kaiser-Meyer-Olkin (KMO). Também, foi realizado o cálculo do Alpha de Cronbach para avaliar a confiabilidade das medidas, onde os construtos apresentaram índices aceitáveis, iguais ou superiores a 0,70 (Malhotra, Birks \& Wills, 2012).

Já a validade convergente dos construtos foi analisada de duas formas: (i) por meio da análise de significância das cargas fatoriais das variáveis, com base nos t-values dos indicadores de cada construto, Ihe conferindo uma significância de $(p<0,05)$; e (ii) pela Análise Fatorial Confirmatória (AFC) (Kline, 2011) (vide Tabela 1).

Tabela 1 - Confiabilidade composta e variância extraída dos construtos

\begin{tabular}{|c|c|c|c|c|}
\hline Construtos & $\begin{array}{c}\text { Variâncias } \\
\text { Explicadas } \\
\text { (\%) }\end{array}$ & $\begin{array}{l}\text { Alpha de } \\
\text { Cronbach }\end{array}$ & $\begin{array}{c}\text { Confiabilidade } \\
\text { Composta }\end{array}$ & $\begin{array}{c}\text { Variâncias } \\
\text { Extraída }\end{array}$ \\
\hline DNS & 71,63 & 0,92 & 0,96 & 0,74 \\
\hline Orientação para o Cliente & 69,24 & 0,91 & 0,91 & 0,72 \\
\hline Competências Profissionais & 82,93 & 0,90 & 0,93 & 0,88 \\
\hline Retenção de Clientes & 74,22 & 0,93 & 0,94 & 0,73 \\
\hline
\end{tabular}

Fonte: Dados provenientes da pesquisa.

Segundo os resultados, percebe-se que a confiabilidade composta para os construtos testados, ficou acima do valor recomendado (acima de 0,70), variando entre 0,91 e 0,96. Em relação à variância extraída, todos os índices foram superiores ao apresentado na literatura, estando entre 0,72 e 0,88

A seguir foi testada a validade discriminante para verificação da distinção entre os construtos testados (Hair Jr. et al., 2014). Diante disso, para a identificação da validade discriminante entre os construtos, foi utilizado o procedimento indicado por Fornell e Larcker (1981), que verifica as correlações entre construtos ao quadrado, conforme Tabela 2. 
Tabela 2 - Validade discriminante

\begin{tabular}{|c|c|c|c|c|}
\hline Construtos & DNS & OC & $\mathrm{CP}$ & Retenção \\
\hline DNS & 0,77 & & & \\
\hline OC & 0,70 & 0,78 & & \\
\hline $\mathrm{CP}$ & 0,59 & 0,60 & 0,84 & \\
\hline Retenção & 0,61 & 0,72 & 0,65 & 0,74 \\
\hline
\end{tabular}

Fonte: Dados provenientes da pesquisa.

Sendo assim, os resultados das análises demonstraram que há validade discriminante entre os construtos, pois a variância extraída e maior que a variância compartilhada.

\subsection{Validação do modelo estrutural}

A partir da estimação do modelo estrutural, iniciou-se a validação do Modelo Teórico, realizada a partir da qualidade das medidas de ajustes (Hair Jr. et al., 2014; Kline, 2015; Byrne, 2016). As medidas de ajustes são baseadas no método de estimação da MLE, que reflete os resultados das análises das matrizes de covariâncias estimadas e observadas, conforme demonstrado na Tabela 3.

Tabela 3 - Medidas de ajustes por construtos

\begin{tabular}{ccccccccc}
\hline \multirow{2}{*}{ Medidas de Ajustes } & GFI & AGFI & RMSEA & TLI & IFI & NFI & CFI \\
\cline { 2 - 8 } & & & & & & & & \\
& 0,884 & 0,843 & 0,066 & 0,938 & 0,951 & 0,911 & 0,950 \\
\hline
\end{tabular}

Fonte: Dados provenientes da pesquisa.

Observando as medidas de ajustes, verifica-se que as medidas $\operatorname{TLI}(0,938)$, IFI $(0,951)$, NFI $(0,911)$ e CFI $(0,950)$ apresentaram medidas de acordo com os índices recomendados na literatura, tendo valores aceitáveis superiores a 0,90. O RMSEA obteve um índice de 0,066, sendo que esta medida de ajustes apresentou o índice acima do recomendado na literatura entre 0,05 e 0,08. Porém, o $\operatorname{GFI}(0,884)$ e o $\operatorname{AGFI}(0,843)$, apresentaram os valores abaixo do recomendado na literatura, que é de 0,90 (Hair Jr. et al., 2010) mas, conforme Bagozzi e Yi (2012), estes índices frequentemente não apresentam valores totalmente aceitáveis. 


\subsection{Teste de hipóteses}

Dando continuidade à validação do modelo, foi realizado o teste de hipóteses, examinando-se a significância e a magnitude dos coeficientes de regressão estimados (Hair Jr. et al., 2014). A Tabela 4 apresenta as hipóteses, os caminhos estruturais, os coeficientes não padronizados, os erros-padrão, os coeficientes padronizados, os t-values e as probabilidades.

Tabela 4 - Teste de hipóteses

\begin{tabular}{cccccccc}
\hline Hi & $\begin{array}{c}\text { Caminhos } \\
\text { Estruturais }\end{array}$ & $\begin{array}{c}\text { Coeficientes Não } \\
\text { Padronizados (b) }\end{array}$ & Erros & $\begin{array}{c}\text { Coeficientes } \\
\text { Padronizados } \\
(\beta)\end{array}$ & $\begin{array}{l}\text { t-values } \\
\text { ( }\end{array}$ & $\mathrm{p}$ & Resultados \\
$\mathrm{H}_{1}$ & $\mathrm{DNS} \rightarrow \mathrm{OC}$ & 0,874 & 0,092 & 0,715 & 9,529 & $\mathrm{p}<0,001$ & Suportada \\
$\mathrm{H}_{2}$ & $\mathrm{OC} \rightarrow \mathrm{RC}$ & 0,463 & 0,076 & 0,659 & 6,098 & $\mathrm{p}<0,001$ & Suportada \\
$\mathrm{H}_{3}$ & $\mathrm{CP} \rightarrow \mathrm{OC}$ & 0,428 & 0,054 & 0,439 & 7,885 & $\mathrm{p}<0,001$ & Suportada \\
$\mathrm{H}_{4}$ & $\mathrm{CP} \rightarrow \mathrm{RC}$ & 0,218 & 0,046 & 0,318 & 4,699 & $\mathrm{p}<0,001$ & Suportada \\
\hline
\end{tabular}

Fonte: Dados descritivos da pesquisa.

Obs: Nível de significância de 0,05.

De acordo com os resultados apresentados, observa-se que as quatro hipóteses iniciais do estudo, foram suportadas estatisticamente, sendo elas a $\mathrm{H}_{1}$ (o DNS influencia positivamente a orientação para o cliente, $\beta=0,715, p<0,001$ ) confirmando os apontamentos de Ludwiczak (2016) e Sindakis (2015), $\mathrm{H}_{2}$ (a orientação para o cliente afeta positivamente a retenção de clientes, $\beta=0,659$, $p<0,001$ ) resultado identificado também por Rao et al. (2011), $\mathrm{H}_{3}$ (a competência profissional afeta positivamente a orientação para o cliente, $\beta=0,439, p=0,015$ ) em congruência com o estudo de Birken et al. (2013), $\mathrm{H}_{4}$ (a competência profissional afeta positivamente a retenção de clientes, $\beta=$ 0,318, p < 0,001) comprovando a indicação de Brach et al. (2015).

Diante dos resultados evidenciados, verifica-se que ao desenvolver um novo serviço, primeiramente, é preciso considerar as particularidades dos serviços profissionais, como é o caso do setor da saúde em estudo, e ao envolvimento dos seus usuários (clientes) dentro do processo de inovação (Sharma, Conduit \& Hill, 2014).

Além disso, verifica-se que as instituições de saúde exigem que seus funcionários, tenham um perfil profissional em constante desenvolvimento para acompanhar as inovações tecnológicas e dos serviços, com potencial para resolução de problemas e pró-atividade, visando desenvolver relacionamentos de longo prazo com os seus clientes (Sindakis, 2015).

Segundo Hair Jr. et al. (2010), outra forma para se verificar a efetividade do teste de hipóteses é por meio dos coeficientes de determinação $\left(R^{2}\right)$ com base nas correlações múltiplas ao quadrado de cada variável dependente. Ao se tratar dos coeficientes de determinação $\left(R^{2}\right)$, verifica-se que o resultado para o modelo estrutural apresentou 71,8\% da variância da Retenção de Clientes (variável 
dependente), que é explicada por suas variáveis independentes, Orientação para o Cliente e a Competência Profissional, conforme apresentados na Tabela 5.

Tabela 5 - Coeficientes de determinação do modelo teórico

\begin{tabular}{cc}
\hline Construtos & Coeficientes de Determinação $\left(R^{2}\right)$ \\
Orientação para o Cliente & 0,703 \\
Competência Profissional & 0,602 \\
Retenção de Clientes & 0,718 \\
\hline
\end{tabular}

Fonte: Dados provenientes da pesquisa.

\subsection{Efeito moderador do tempo de cliente entre a orientação para o cliente e a retenção de clientes}

Na realização do teste de moderação foi analisado o tempo como cliente da operadora de plano de saúde dos respondentes, a qual foi realizada por meio da análise multigrupos (Hayes, 2013), dividindo a amostra em dois grupos, sendo o Grupo 1 com tempo de cliente baixo e o Grupo 2 com tempo de cliente elevado. Para a realização dos testes comparativos entre os grupos, utilizou-se o teste de Índices Críticos para Diferenças entre os Parâmetros (Critical Ratios for Differences Between Parameters). Para tanto, foi utilizado o critério das diferenças entre os grupos com Z-scores, os quais devem ser superiores a $\pm 1,96$, indicando um nível de significância menor do que $0,05(p<0,05)$ e um intervalo de confiança de 95\% (Preacher \& Hayes, 2004; Zhao, Lynch Jr. \& Chen, 2010).

A análise testou o efeito moderador da idade na relação entre os construtos Orientação para o Cliente (OC) e Retenção de Clientes (RC). A Tabela 6 apresenta os resultados do efeito da moderação.

Tabela 6 - Efeito moderador do tempo de cliente na relação entre OC e RC

\begin{tabular}{cccccccc}
\hline & $\begin{array}{c}\text { Tempo de } \\
\text { Cliente } \\
\text { (anos) }\end{array}$ & $\begin{array}{c}\text { Coeficientes Não } \\
\text { Padronizados (b) }\end{array}$ & $\begin{array}{c}\text { Erros- } \\
\text { Padrão }\end{array}$ & $\begin{array}{c}\text { Coeficientes } \\
\text { Padronizados ( } \beta \text { ) }\end{array}$ & t-values & $p$ & Z-Scores \\
\hline & 1 a 5 & 0,310 & 0,092 & 0,488 & 4,983 & $p=0,001$ & $-2,241$ \\
$H_{5}$ & 6 a 10 & 0,548 & 0,048 & 0,623 & 9,498 & $p<0,001$ & \\
\hline
\end{tabular}

Fonte: Dados provenientes da pesquisa.

No teste da $\mathrm{H}_{5}$, verificou-se que a relação entre os construtos Orientação para o Cliente e a Retenção de Clientes, na percepção dos respondentes, é potencializada junto ao Grupo 2 (6 a 10 anos de cliente), indicando que a Orientação para o Cliente tem um efeito positivo e mais forte sobre a Retenção de Clientes para aqueles clientes com mais tempo de contrato $(b=0,548, p<0,001)$, se comparados com os do Grupo 1 ( 1 a 5 anos de cliente), clientes mais recentes ( $b=0,310, p=0,001$ ). 


\section{Considerações finais}

Tendo como ponto de partida a abordagem dos estudos empíricos que relacionaram os construtos contemplados, um novo modelo teórico foi proposto e testado. Em termo das hipóteses de pesquisa, as contribuições teórico-empíricas, estão relacionadas com a confirmação das hipóteses que contemplaram os construtos orientação para o cliente, competências profissionais e retenção de clientes como consequentes do Desenvolvimento de Novos Serviços, as quais compreenderam o Modelo Teórico proposto, testado e validado, no contexto de operadoras de planos de saúde.A primeira contribuição do estudo, portanto, foi a confirmação da relação positiva e direta do DNS com a Orientação para o Cliente $\left(\mathrm{H}_{1}\right.$ : DNS $\left.\rightarrow \mathrm{OC}\right)$. Os resultados encontrados convergem com a literatura, poisalguns estudos consideraram uma relação positiva entre o DNS e a Orientação para o Cliente, com o intuito de oferecer soluções personalizadas e criativas aos clientes (Wong \& Tong, 2012), partindo do princípio que, as empresas que se preocupam e envolvem seus clientes no DNS são mais propensas a ter maior sucesso no mercado onde atuam (Sindakis, 2015).

Neste sentido, é importante destacar que a área da saúde apresenta particularidades decorrentes da necessidade de constituir vínculos entre os sistemas nacionais de inovação e o bemestar social (Costa, 2016). Em se tratando do sistema de saúde supementar, a qual é composta por uma cadeia de atividades que faz a ligação entre consumo de recursos e usuários finais (Almeida, \& Guerra, 2019), observa-se que, com a mercantilização da saúde, as operadoras de saúde privadas no Brasil ganham a cada dia maior espaço de mercado.

Destaca-se, ainda, que o estudo ainda traz como contribuição a confirmação da relação entre o impacto positivo da Orientação para o Cliente sobre a Retenção de Clientes $\left(\mathrm{H}_{2}: \mathrm{OC} \rightarrow \mathrm{RC}\right)$, demonstrando que a orientação para o cliente afeta as trocas relacionais em uma perspectiva de longo prazo. Este resultado encontrado se diferencia do estudo desenvolvido por Guo e Wang (2015) que apresentou um efeito indireto entre a orientação para o cliente e a retenção de clientes, considerando aumento na lucratividade da empresa.

Outra contribuição proveniente, é a confirmação de que as Competências Profissionais influenciam direta e positivamente a Orientação para o Cliente $\left(\mathrm{H}_{3}: \mathrm{CP} \rightarrow \mathrm{OC}\right)$ e, da mesma forma,a Retenção de Clientes $\left(\mathrm{H}_{4}: \mathrm{CP} \rightarrow \mathrm{RC}\right)$. A relevância de tal contribuição é apontada, também, por Frey, Bayón e Totzek (2013), os quais salientam que a busca pela satisfação e retenção de clientes deve ser um objetivo primordial para os funcionários, pois a retenção de clientes influencia diretamente na retenção dos funcionários.

Para implementar efetivamente as inovações em serviços de saúde, é necessário que os funcionários tenham informações sobre o que fazer, como e quando fazê-las e porque devem fazer, primando para a eficácia para implemantar a inovação que novos serviços podem trazer para as operadoras de serviços de saúde (Bokhour et al., 2018). 
Diante dos resultados do presente estudo, percebe-se que as mudanças que estão ocorrendo no mercado impactam também no nível de exigências dos clientes em relação à qualidade dos serviços que lhe são oferecidos na área da saúde. Dessa forma, os prestadores de serviços da saúde precisam estimular a inovação dos serviços da saúde visando melhorias contínuas (Nodari, Olea \& Dorion, 2013). Além disso, o nível de exigência dos pacientes e o volume de informações a que eles têm acesso fazem com que as instituições de saúde busquem cada vez mais formas, de satisfazer as expectativas e necessidades dos seus clientes (Sindakis, 2015).

Ao se tratar das implicações gerenciais, verifica-se que a necessidade de envolvimento do cliente como co-produtor no processo de DNS e em inovações em serviços, em uma perspectiva de longo prazo, que vise a criação de um ambiente mais dinâmico e competitivo. Isto implica, que as operadoras de planos de saúde precisam desenvolver novos serviços orientados para os clientes, e para isso, precisam investir em novas tecnologias e ter equipes melhor treinadas e qualificadas, para que possam oferecer serviços de qualidade superior à concorrência, aproximando-se cada vez mais de seus clientes de forma lucrativa e rentável.

Neste contexto, sugere-se, ainda, o desenvolvimento de novas competências na gestão e nas equipes, por meio de mudanças dos processos ou procedimentos, com sistemas informatizados, novas ferramenta de gestão e configurações institucionais, que permitam um maior envolvimento do clientesjunto às operações das empresas, tendo a intenção de aprimorar os elementos que impactam no processo de inovação dos serviços de saúde.

Como desenvolvimento de pesquisas futuras, sugere-se testar outros construtos como inovação em serviços, qualidade percebida, satisfação de clientes, valor de uso dos serviços, , confiança, continuidade de uso de serviços e lealdade de clientes. Além disso, os modelos teóricos propostos, permitindo a comparação entre modelos rivais ou modelos aninhados, os quais poderiam ser testados em outros contextos de serviços (financeiro, turísticos e educacionais) com a intenção de aprimorar a precisão científica dos construtos analisados, , gerando evidência mais robustas e instigando implicações gerenciais relevantes, levando em consideração que o DNS, impacta sobremaneira na performance das organizações que operam no setor de serviços, nas mais diversos segmentos ou nichos de mercado.

\section{Referências}

Almeida, D. C. R., \& Guerra, L. (2019). Custos e formação de preços nas operadoras de planos de saúde no Brasil. Journal of Health Care Management \& Primary, 11, 1-2.

Amidu, A., \& Aluko, B. T. (2007). Client influence in residential property valuations: an empirical study. Property Management, 25(5), 447-461.

ANS - Agência Nacional de Saúde Suplementar. (2019). Dados e indicadores do setor: beneficiários de planos privados de saúde. Disponível em: 
http://www.ans.gov.br/images/stories/Materiais_para_pesquisa/Perfil_setor/Dados e indicadores d o setor/total-cad-info-jun-2019.pdf.

Arnold, T. J., Fang, E., \& Palmatier, R. W. (2011). The effects of customer acquisition and retention orientations on a firm's radical and incremental innovation performance. Journal of the Academic Marketing Science, 39, 234-251.

Bagozzi, R. P., \& Yi, Y. (2012). Specification, evaluation, and interpretation of structural equation models. Journal of the Academic Marketing Science, 40, 8-34.

Biemans, W. G., Griffin, A., \& Moenaert, R. K. (2016). New service development: how the field developed, its current status and recommendations for moving the field forward. Journal of Product Innovation Management, 33(4), 382-397.

Birken, S. A., Lee, S. D., Weiner, B. J., Chin, M. H., \& Schaefer, C. T. (2013). Improving the effectiveness of health care innovation implementation: middle managers as change agents. Medical Care Research and Review, 70(1), 29-45.

Bokhour, B. G., Griffin, A., Fix, G. M., Mueller, N. M., Baker, A. M., Lavela, S. L., \& Hill, J. N. (2018). How can healthcare organization implemented patiente-centered care? Examining a large-scale cultural transformation. BMC Health Services Research, 18(1), 1-11.

Brach, S., Walsh, G., Hennig-Thurau, T., \& Groth, M. (2015). A dyadic modelo of customer orientation: mediation and moderation effects. British Journal of Management, 26, 292-309.

Bruno, A., Dell'Aversana, G., \& Zunino, A. (2017). Customer orientation and leardership in the health service sector: the role on workplace social support. Frontiers in Psychology, 8, 1-6.

Burton, J., Story, V. M., Raddats, C., \& Zolkiewski, J. (2017). Overcoming the challenges that hinder new service development by manufactures with diverse services strategies. International Journal of Production Economics, 192(C), 29-39.

Byrne, B. M. (2016). Structural equation modeling with AMOS: basic concepts applications, and programming. $3^{\text {rd }}$ edition. New York: Routledge.

Carbonell, P., \& Rodriguez-Escudero, A. I. (2014). Antecedents and consequences of using information from customers involved in New Service Development. Journal of Business and Industrial Marketing, 29(2), 112-122.

Costa, L. S. (2016). Inovação nos serviços de saúde: apontamentos sobre os limites do conhecimento. Cadernos de Saúde Pública, 32, 51-62.

Dagger, T. S., \& David, M. E. (2012). Uncovering the real effect of switching costs on the satisfactionloyalty association: the critical role of involvement and relationship benefits. European Journal of Marketing, 36(3/4), 447-468.

Días, G. R. (2017). The influence of satisfaction on customer retention in mobile phone market. Journal of Retailing and Consumer Services, 36, 75-85.

Dominguez-Péry, C., Ageron, B., \& Neubert, G. (2013). A service science framework to enhance value creation in service innovation projects: an RFID case study. International Journal of Production Economics, 141(2), 440-451. 
Edvardsson, B., Kristensson, P., Magnusson, P., \& Sundström, E. (2012). Customer integration in service development and innovation - methods and a new framework. Technovation, 32(7/8), 419-429.

Fornell, C., \& Larcker, D. (2013). Evaluating structural equation models with unobserved variables and measurement error. Journal of Marketing Research, 18(1), 39-50.

Framback, R. T., Fiss, P. C., \& Ingenbleek, P. T. M. (2016). Important is customer orientation for firm performance? A fuzzy set analysis of orientations, strategies, and environments. Journal of Business Research, 69, 1.428-1.436.

Frey, R., Bayón, T., \& Totzek, D. (2013). How customer satisfaction affects employee satisfaction and retention in a professional services context. Journal of Service Research, 16(4), 503-517.

Gebauer, H., Gustafsson, F., \& Witell, L. (2011). Competitive advantage through service differentiation by manufacturing companies. Journal of Business Research, 64(12), 1.270-1.280.

Graciola, A. P., Bebber, S., Eberle, L., Rizzon, F., \& Milan, G. S. (2017). A comparative analysis of new service development: two periods of research. European Journal of Economics, Finance and Administrative Science, 93, 114-130.

Gremyr, I., Witell, L., Löfberg, N., Edvardsson, B., \& Fundin, A. (2014). Understanding new service development and service innovation through innovation modes. Journal of Business, Industry Marketing, 29(2), 123-131.

Groth, M., Hennig-Thurau, T., \& Walsh, G. (2009). Customer reactions to emotional labor: the roles of employee acting strategies and customer detection accuracy. Academy of Management Journal, 52, 958-974.

Guo, C., \& Wang, Y. (2015). How manufacture market orientation influences B2B customer satisfaction and retention: empirical investigation of the three market orientation components. Journal of Business \& Industrial Marketing, 30(2), 182-193.

Hair, Jr. J. F., Black, W. C., Babin, B. J., \& Anderson, R. E. (2010). Multivariate data analysis. $7^{\text {th }}$ edition. New York: Prentice Hall.

Hair J. J. F., Hult, G. T. M., Ringle, C. M., \& Sarstedt, M. (2014). A Primer on partial least squares structural equation modeling (PLS-SEM). Los Angeles: Sage Publications.

Hayes, A. F. (2013). Introduction to mediation, moderation, and conditional process analysis: a regression-based approach. New York: The Guilford Press.

Hennig-Thurau, T. (2004). Customer orientation of service employees: Its impact on customer satisfaction, commitment, and retention. International Journal of Service Industry Management, 15, 460-478.

Hillebrand, B., Nijholt, J. J., \& Nijssen, E. J. (2011). Exploring CRM effectiveness: an institutional theory perspective. Journal of the Academy of Marketing Science, 39, 592-608.

Hu. M. M., Horng, J., \& Sun, Y. C. (2009). Hospitality teams: knowledge sharing and service innovation performance. Tourism Management, 30, 41-50.

Jaakkola, E., Meiren, T.,Witell, L., Edvardsson, R., Schäfer, A., Reynoso, J., Sebastiani, R., \& Weitlaner, D. (2017). Does one size fit all? New service development across different types of services. Journal of Service Management, 28(2), 329-347. 
Jong, J. P. J., \& Vermeulen, P. A. M. (2003). Organizing successful new service development: a literature review. Management Decisions, 41(9), 844-858.

Kim, N., \& Atuahene-Gima, K. (2010). Using exploratory and exploitative market learning for new product development. The Journal of Product Innovation Management, 27(4), 519-536.

Kline, R. B. (2015). Principles and practice of structural equation modeling. $4^{\text {th }}$ edition. The Guilford Press. New York: London.

Kohli, A. K., \& Jaworski, B. J. (1990). Market orientation: the construct, research, and managerial implications. Journal of Marketing, 54(2), 1-18.

Kuester, S., Schuhmacher, M. C., Gast, B., \& Worgul, A. (2013). Sectoral heterogeneity in new service development: an exploratory study of service types and success factors. Journal of Product Innovation Management, 30(3), 533-544.

Ludwiczak, A. (2016). Customer requirements and the quality improvement of health care services. Management, 20(2), 428-441.

Malhotra, N. K., Birks, D., \& Wills, P. (2012). Marketing research: applied approach. $4^{\text {th }}$ edition. New York: Pearson.

Marquardt, A. J., Golicic, S. L., \& Davis, D. F. (2011). B2B services branding in the logistics services industry. Journal of Service Marketing, 25(1), 47-57.

McCoy, M. A., Levett-Jones, T., \& Pitt, V. (2013). Development and psychometric testing of the ascento to competence scale. Nurse Education Today, 33, 15-23.

Milan, G. S., Eberle, L., \& Bebber, S. (2015). Perceived value, reputation, trust and switching costs as determinants of customer retention. Journal of Relationship Marketing, 14, 109-123.

Neto, M. T. R., Souza, J., \& Souki, Q. (2011). Identifying variables that predict clients propensity to end their checking accounts. International Journal of Bank Marketing, 29(6), 489-507.

Niedenthal, P. M., \& Brauer, M. (2012). Social functionality of human emotions. Annual Review of Psychology, 63, 259-285.

Nodari, C. H., Olea, P. M., \& Dorion, E. C. H. (2013). Relação entre inovação e qualidade da orientação do serviço de saúde para atenção primária. Revista de Administração Pública, 5(47), 1243-264.

O'Cass, A., Song, M., \& Yuan, L. (2013). Anatomy of service innovation: introduction on the special issue. Journal of Business Research, 66(8), 1.060-1.062.

Ordanini, A., \& Parasuraman, A. P. (2011). Service innovation viewed through a service-dominant logic lens: a conceptual framework and empirical analysis. Journal of Service Research $\_$14(1), 43-23.

Papastathopoulou, P., \& Hultink, E. J. (2012). New service sevelopment: sn analysis of 27 years of research. Journal of Product Innovation Management, 29(5), 705-714.

Preacher, K. J., \& Hayes, A. F. (2004). Asymptotic and resampling strategies for assessing and comparing indirect effects in simple and multiple mediator models. Behavior Research Methods, 40(3), 879-889. 
Rao, S., Goldsby, T. J., Griffis, S. E., \& Iyengar, D. (2011). Electronic logistics service quality (e-LSQ): its impact on the customer's purchase satisfaction and retention. Journal of Business Logistics, 32(2), 167-179.

Sampaio; C. H., Perin, M. G., \& Ferreira, G. C. (2008). A relação entre sucesso de novos produtos, orientação para o mercado e performance empresarial. Produto \& Produção, 9(3), 85-94.

Santos, J. B., \& Spring, M. (2013). New service development: managing the dynamic between services and operations resources. International Journal of Operating \& Production Management, 33(7), 800827.

Santos-Vijande, M. L. S., López-Sánchez, J. A., \& Rudd, J. (2016). Frontline employees collaboration in industrial service innovation: routes of co-creation's effects on new service performance. Journal of the Academic Marketing Science, 44, 350-375.

Sharma, S., Conduit, J., \& Hill, S. R. (2014). Organisational capabilities for customer participation in health care service innovation. Australian Marketing Journal, 22(3), 179-188.

Sindakis, S. (2015). Corporate venturing and customer-driven innovation in the mental health-care market: a review of the literature and development of a conceptual framework. Journal of the Knowledge Economy, 6(4), 1.013-1.033.

Storey, C., \& Hull, F. M. (2010). Service development success: a contingent approach by knowledge strategy. Journal of Service Management, 21(2), 140-161.

Tajeddini, K. (2011). Customer orientation, learning orientation, and new service development and empirical investigation of the Swiss hotel industry. Journal of Hospitality \& Tourism Research, 35(4), 437-468.

Wang, Q., Zhao, X., \& Voss, C. (2016). Customer orientation and innovation: a comparative study of manufacturing and service firms. International Journal Production Economics, 171, 211-230.

Witell, L., Gebauer, H., Jaakkola, E., Hammedi, W., Patricio, L., \& Perks, H. (2017). A bricolage perspective on service innovation. Journal of Business Research, 79, 290-298.

Wong, S. K. S., \& Tong, C. (2012). The influence of market orientation on new product success. European Journal of Innovation Management, 15(1), 99-121.

Wu, Y., Tsai, C., Hsiung, H., \& Chen, K. (2015). Linkage between frontline employee service competence scale and customer perceptions of service quality. Journal of Services Marketing, 29(3), 224-234.

Zhao, X., Lynch Jr., J. G., \& Chen, Q. (2010). Reconsidering Baron and Kenny: myths and truths about mediation analysis. Journal of Consumer Research, 37(2), 197-206.

Recebido em: 19 dez. 2019 / Aprovado em: 23 fev. 2020

Para referenciar este texto

American Psychological Association (APA)

Eberle, L., \& Milan, G. S. (2020, out./dez.). Desenvolvimento de Novos Serviços, orientação para o cliente, competências profissionais e retenção de clientes em serviços de saúde. Exacta, 18(4), 708-724. https://doi.org/10.5585/exactaep.v18n4.16274. 